\title{
Simulation of Fuzzy Control System for Motor Mechanism of Coal Mine Disconnector Breaking Test
}

\author{
Jianfeng Zhu \\ CCTEG Shenyang Research Institute, Fushun, Liaoning, 113122, China \\ zhang651779986@126.com
}

Keywords: Coal mine disconnector, Motor mechanism, Curve setting, Fuzzy PID control.

\begin{abstract}
In order to improve the controllability of coal mine disconnector breaking tests and realize intelligent operation, this paper put forward a simulation of fuzzy PID control system for motor mechanism of coal mine disconnector breaking test. The simulation model of motor mechanism speed, position, and three closed-loop control system of torque is established by using the Simulink simulation platform in MATLAB and combining the designed PID controller and fuzzy PID controller. And finished by aiming at each sub module in the simulation model and based on the coal mine disconnector breaking test process curve. The results show that compared with the conventional PID control, the fuzzy PID control has the advantages of fast response time, high stability and good tracking effect, and is more suitable for the intelligent operation of coal mine disconnector operation.
\end{abstract}

\section{Introduction}

With the development of wind, solar and biomass energy technology and the utilization of clean coal technology is not yet mature, the current coal industry market is bleak, but the characteristics of energy in China is rich in coal, lean oil and less gas, which determines the coal is still the main body of the energy structure. It is the inevitable trend of the development of coal mine by strengthening the mine safety production management, eliminating the hidden trouble, optimizing the industry structure of coal industry, promoting the safe, efficient, green and intelligent exploit and clean, efficient, low carbon and intensive utilization of coal mine. Breaking test is an important item of approval inspection of coal mine disconnector related products, and is an important index to measure the product quality and safety performance.

\section{Coal Mine Disconnector}

Coal mine disconnector is an important component of electrical equipment, such as mining flameproof vacuum electromagnetic starter, the integrated protection device of mining flameproof electric coal drill transformer, mining flameproof multi loop combination switch and mining flameproof and intrinsically safe soft starter. It plays a vital role in cut off the fault current, electrical isolation and no load reversing in coal mine power system. The GHK400/1140V coal mine disconnector is the research object in this paper, the physical map is shown in figure 1.

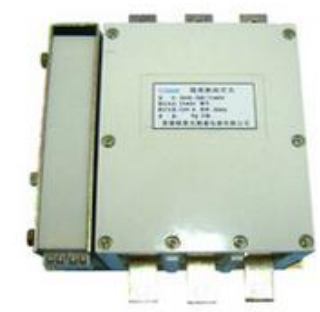

(a) Top-down view

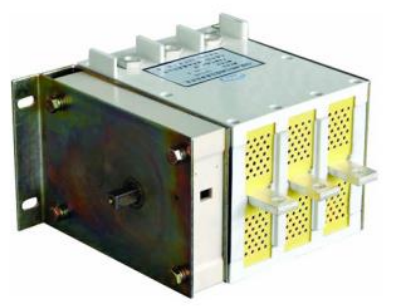

(b) Side view

Fig1. The physical map of GHK400/1140V coal mine disconnector

The traditional breaking test operating mechanism is divided into the manual operating mechanism and the pneumatic operating mechanism due to the coal mine disconnector itself does not has the operating mechanism,. The manual operation mechanism test requires the cooperation of two people 
and after switching on test current about $100 \mathrm{~ms}$, they should pull the rope quickly and drive coal mine disconnector revolving shaft to complete coal mine disconnector breaking test. This kind of operating mode has the disadvantages of poor stability, too dependent on the experimental personnel experience, and has security risks. The breaking test of coal mine disconnector pneumatic operating mechanism is finished by rotation control of swing cylinder and driving the action of shaft. This mode of operation uses mechanical device to replace manual operation, which can ensure the automation of breaking operation, but the test device has complex structure, large total exercise tolerance and low stability, poor controllability, and cannot realize the accurate adjustment of breaking velocity and time.

The motor mechanism drives the rotating shaft, the operating disk, the coaxial four link mechanism, the operating arm and so on to realize the coal mine disconnector moving contact completing the breaking test. The motor has the advantages of simple structure, high energy utilization rate (electrical energy convert into mechanical energy), good safety, and providing technical support for the pilot test and approval inspection of coal mine disconnector and related products, realizing the controllable and intelligent operation of the whole test process.

\section{Control System Simulations}

\subsection{Modeling of Control System}

In the simulation environment of MATLAB Simulink and using the method of modular, the motor mechanism of coal mine disconnector breaking test control system is divided into several functional modules and modeling respectively. The simulation model of the whole system is established through the organic combination of these modules. Motor module is used for the calculation of inside motor parameters. Commutation module is used for detecting the rotor position of the motor through Hall signal, and providing driving signals for the break over and shutoff of IGBT module. The energy supply and regulator module are composed of controlled voltage source and IGBT, it is used for power supply for three-phase winding of motor. Speed loop is adjusted by fuzzy PID or conventional PID controller, and position loop is adjusted by PI controller. The principle schematic of control system simulation is shown in figure 2 .

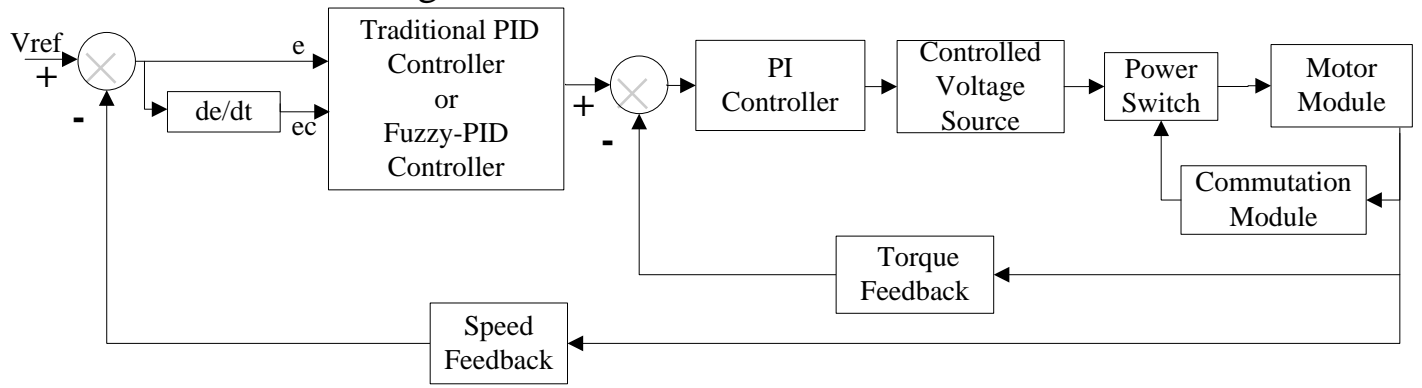

Fig.2 Principle schematic of motor mechanism control system of coal mine disconnector breaking test

\subsection{PID Control Simulation}

According to the operation process of coal mine disconnector breaking test, the motor control system is modeled. The control system is realized through the speed and torque double closed loop, the outer speed loop adopts the conventional PID control algorithm, and the inner torque loop adopts PI control algorithm. The outer loop speed regulation is achieved by error signal through the PID controller. The output signal of speed loop is used as the input signal of torque PI controller, and the output signal of the torque loop is used to provide control signal for controllable voltage source. Then, the motor is driven by IGBT to drive the contacts for processing the breaking test.

The final ideal result can be obtained through simulation analysis repeatedly and the overall adjustment and fine adjustment of control system parameters, as shown in Fig. 3. The results show that the system has large errors in the later. The fuzzy PID controller in the outer loop speed regulator system is introduced to replace the traditional PID controller in the original system, expecting to improve the tracking effect through further improvement of control algorithm. 


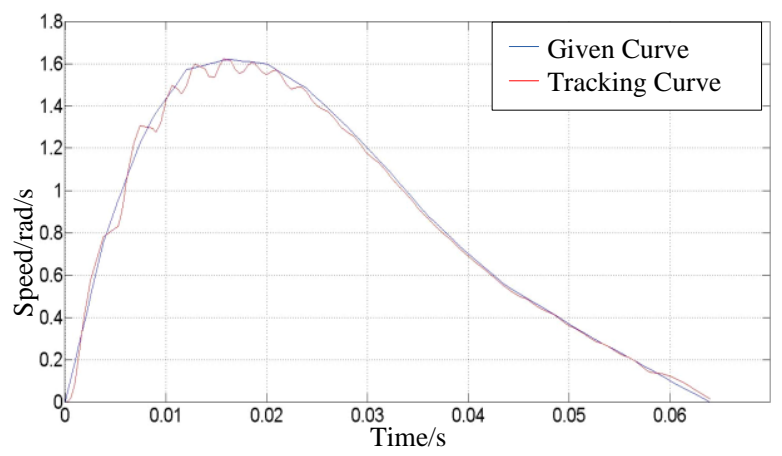

Fig.3 Traditional PID controller speed tracking control simulation curve

The final ideal result can be obtained through simulation analysis repeatedly and the overall adjustment and fine adjustment of control system parameters, as shown in Fig. 5. The results show that the system has large errors in the later. The fuzzy PID controller in the outer loop speed regulator system is introduced to replace the traditional PID controller in the original system, expecting to improve the tracking effect through further improvement of control algorithm.

\subsection{Fuzzy PID control simulation}

It is very difficult to describe and control the speed curve of breaking tests by precise mathematical model on account of the complexity of coal mine disconnector breaking test and the diversity of load. However, the fuzzy adaptive PID controller takes the deviation e and deviation change rate ec as inputs, which can meet the demand of PID parameters self-tuning of e and ec at different moments. The working process of the fuzzy controller can be described as: firstly, the fuzzification of the input e and ec of controller. Secondly, fuzzy logic decision based on fuzzy control rules. The fuzzy output of controller is obtained by using fuzzy logic reasoning algorithm. Finally, the exact values obtained by precision are the three parameters of PID controller, respectively. The fuzzy and PID hybrid control strategy not only retains the fast response characteristics of fuzzy controller, but also introduces the PID control algorithm, which will greatly improve the stability of the system.

Taking velocity error e and error change rate ec as input, coefficient $\mathrm{KP}, \mathrm{KI}, \mathrm{KD}$ as output to construct two-dimensional fuzzy controller. The fuzzy linguistic variables $\mathrm{E}$ and Ec can be obtained after fuzzification process of the deviation of input e and the change rate of deviation ec. The fuzzy subsets of E and Ec were $\{$ NB, NM, NS, Z, PS, PM, PB $\}$. Similarly, the fuzzy subsets of KP, KI, and $\mathrm{KD}$ are $\{\mathrm{NB}, \mathrm{NM}, \mathrm{NS}, \mathrm{Z}, \mathrm{PS}, \mathrm{PM}, \mathrm{PB}\}$. On the one hand, the fuzzy linguistic variables and the domain of discourse $\{-6,-5,-4,-3,-2,-1,0,1,2,3,4,5,6\}$ of $\mathrm{KP}, \mathrm{KI}$, and KD were selected. On the other hand, supposing that $\mathrm{E}$ and Ec both obey the distribution of Gauss membership function curve, $\mathrm{KP}, \mathrm{KI}$ and KD all obey the distribution of triangle membership function curve. The membership function of input and output is shown in figure 4.

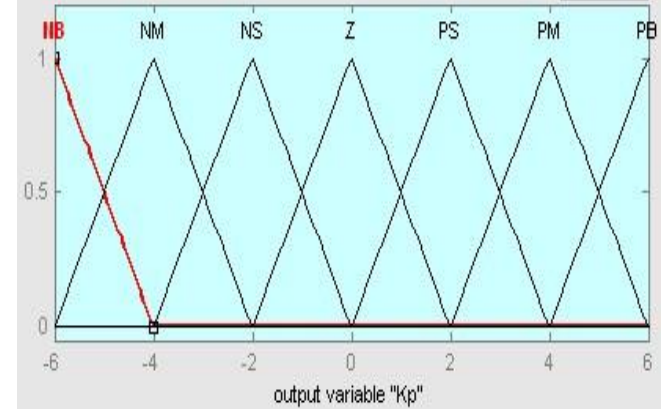

(a) Output ratio coefficient $\mathrm{Kp}$

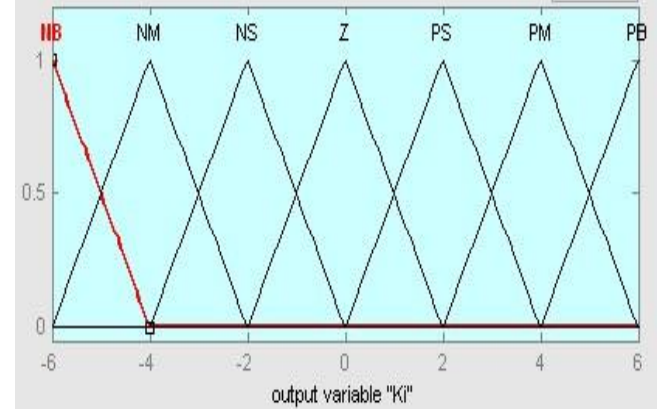

(b) Output integral coefficient Ki

Fig.4 The function of output membership

Fuzzy control rule is a set of fuzzy conditional statements derived from expert experience and operator skills. The fuzzy rule base of this model can be described by the following 49 fuzzy conditional statements:

(1) If (e is $\mathrm{NB}$ ) and (ec is $\mathrm{NB}$ ) then (Kp is $\mathrm{PB})(\mathrm{Ki}$ is $\mathrm{NB})(\mathrm{Kd}$ is $\mathrm{PS})$

(2) If (e is $\mathrm{NB}$ ) and (ec is $\mathrm{NM}$ ) then (Kp is $\mathrm{PB})(\mathrm{Ki}$ is $\mathrm{NB})(\mathrm{Kd}$ is $\mathrm{PS})$ 
(49) If (e is $\mathrm{PB})$ and (ec is $\mathrm{PB})$ then $(\mathrm{Kp}$ is $\mathrm{NB})(\mathrm{Ki}$ is $\mathrm{PB})(\mathrm{Kd}$ is $\mathrm{PB})$

The precise calculation is carried at last in order to make the fuzzy quantity of the linguistic expression back to the exact value, which is to calculate the output value according to the membership degree of the output fuzzy subset. This paper adopts gravity method for accurate calculation to obtain the KP, KI, KD correction value Fuzzy (KP), Fuzzy (KI), Fuzzy (KD).

Simulation research on fuzzy controller was carried out by using the fuzzy system in Matlab to develop toolbox. After debugging of fuzzy adaptive PID controller, it was imported into the motion control system of motor actuator, and through further fine tuning of the proportion factor and quantization factor in fuzzy controller, speed tracking curve of motor mechanism for coal mine disconnector breaking test was obtained at last, as shown in figure 5. The simulation results show that the system response speed and tracking accuracy have been greatly improved, and the setting defects of PID parameters in conventional PID controller was improved, thus, leading to better completion of speed tracking of motor mechanism for coal mine disconnector breaking tests.

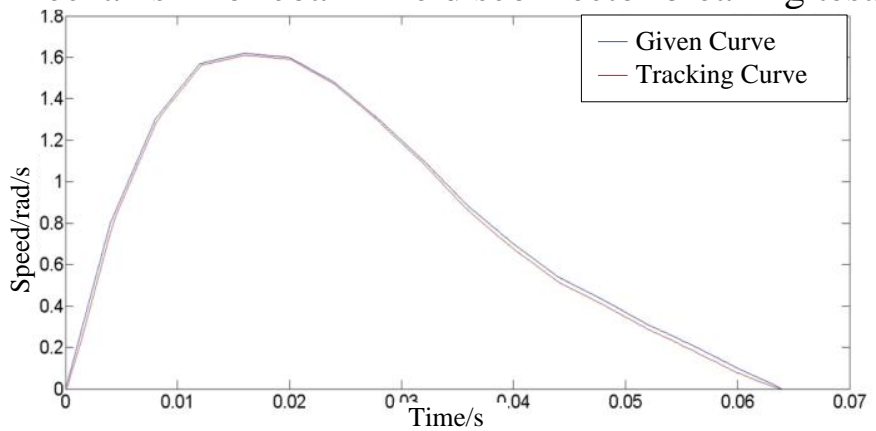

Fig.5 the results of fuzzy PID controller speed tracking control simulation

\section{Conclusion}

In summary, the modeling and simulation analysis of the motor mechanism control system for coal mine disconnector breaking test is carried out. On the one hand, the fuzzy PID controller overcomes the influence of parameter selection and improves the reliability and precision of speed tracking. On the other hand, compared with the simulation results of the traditional PID controller, it is proved that the fuzzy adaptive PID controller is more suitable for the speed tracking control of motor mechanism for the coal mine disconnector breaking test.

\section{References}

[1]. GENG Wei, LU Yong. The cause analysis and treatment of a inner discharge case caused by moistened 35kV switchgear. Electric Switchgear, No.4, 65-66,2008(4).

[2]. YAN Qun. Design and analysis of insulation structure of solid insulation switchgear. Mechanical Research \& Application, No.5,77-80,2009.

[3]. Xin Lin, Huijun Gao, Jianyuan Xu. The calculation and analysis of the coupled problem in the permanent magnetic actuator for vacuum circuit breaker. Digests of the 9th Biennial IEEE Conference on Electromagnetic Field Computation (CEFC)296,2000.

[4]. HAN She-jiao, MA Xi-kui, GUO Guo-ling. Electrical fields computation of tank type metal-oxide surge arrester for 110kV GIS. Insulators and Surge Arresters, No.1,33-35,2002.

[5]. DANG Zhen-ping, PEN Zong-ren. Errosion test on 500kV composite rod insulator fractured in service and its electric tress estimation. Insulators and Surge Arresters, No.4,1-7,2005.

[6]. GAO Bo, ZHANG Ya-ting, WANG Qing-liang, et al. Effect of pollution non-uniformity on electric field of contaminated insulator. Insulators and Surge Arresters, No3,13-16,2008. 